\title{
Resiliencia y satisfacción laboral en profesores de colegios municipales y particulares subvencionados de la comuna de Machalí
}

\author{
Resilience and job satisfaction among teachers from public \\ and private subsidized schools from the commune of Machalí
}

Camila Díaz Sánchez, ${ }^{a}$ Enrique Barra Almagiá ${ }^{b}$

aUniversidad Católica del Maule

Telf.: (56) 42780477. Correo electrónico: ucm.psico.camiladiaz@gmail.com

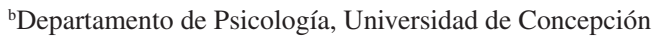

Telf.: (56) 412133614. Correo electrónico: ebarra@udec.cl

\begin{abstract}
El presente estudio evaluó la relación entre resiliencia y satisfacción laboral en un grupo de 119 profesores perteneciente a colegios municipales y particulares subvencionados de la Comuna de Machalí, VI región, Chile. Los resultados obtenidos indican que el nivel de resiliencia es relativamente alto, en cambio el nivel de satisfacción laboral es menor. Se encontró una relación positiva significativa entre resiliencia y satisfacción laboral en la muestra de profesores estudiados, lo cual indicaría que las características disposicionales de la resiliencia serían factores que permiten, en alguna medida, que los profesores deriven satisfacción de su desempeño laboral a pesar del complejo escenario en el cual desempeñan su rol docente.
\end{abstract}

Palabras clave: profesores, resiliencia, satisfacción laboral.

\section{ABSTRACT}

This study examined the relationship between resilience and job satisfaction in a group of 119 teachers belonging to public and private-subsidized schools from Machalí, Chile. The results indicate that the level of resilience is relatively high, whereas the level of job satisfaction is lower. A significant positive relationship between resilience and job satisfaction in the sample of teachers studied was found, this would indicate that the dispositional characteristics of resilience are factors which allow that, at some degree, teachers derive satisfaction from their job performance despite the complex scenario in which they play their teaching role.

Key words: teachers, resilience, job satisfaction.

Esta investigación ha sido elaborada gracias al financiamiento de la Comisión Nacional de Investigación Científica y Tecnológica (CONICYT-PCHA), beca de magíster nacional año 2013, 22131562. 


\section{INTRODUCCIÓN}

La psicología de la educación orienta su campo de estudio y temas de investigación hacia el proceso de enseñanza aprendizaje, considerando las múltiples variables involucradas. Dentro de este proceso el profesor sería un factor clave, ya que el elemento central de la calidad educativa es lo que sucede dentro de la sala de clases, en especial el comportamiento del docente (Arancibia, Herrera \& Strasser, 2008).

Sin embargo, muchas veces los profesionales de la educación se encontrarían en una situación de riesgo en lo que concierne a su salud mental por los diversos factores sociales, políticos y culturales que afectan directamente la dinámica de la sala de clases. Ellos deben enfrentar múltiples desafíos y adaptarse constantemente a desempeñar su rol en un contexto inmerso en la multiculturalidad, globalización y nuevas tecnologías, dimensiones claves en la sociedad del conocimiento. Esta situación de riesgo del profesorado amenaza el proceso educativo, su salud física y psicológica, dejando en evidencia múltiples consecuencias: frágil salud mental, desgaste profesional, estrés y burnout (Claro \& Bedregal, 2003; Corvalán, 2005; Hermosa, 2006). Sin embargo, no todos los profesores se verían afectados por las condiciones adversas en que deben desempeñar su rol, ya que algunos saldrían fortalecidos y mostrarían conductas y actitudes saludables pese a los requerimientos.

Esta idea de desarrollar capacidades aun en situaciones adversas se inscribe en la nueva Psicología Positiva, donde emerge el concepto de resiliencia relacionado a los aspectos positivos presentes en las personas, que actúan como un amortiguador para el estrés y eventos negativos, contribuyendo así a la calidad de vida (Fiorentino, 2008). El concepto de resiliencia se concibe como la capacidad que tienen las personas para enfrentar y sobreponerse a las adversidades y obstáculos que aparecen en sus vidas, y a la vez proyectarse y conseguir el logro de metas propuestas (Saavedra, 2011).

El propósito de la presente investigación fue conocer la influencia de la resiliencia sobre la satisfacción laboral en profesores de la comuna de Machalí, con el objetivo de determinar si esta capacidad es un factor clave que permite que los profesores mejoren la percepción de bienestar asociada a la satisfacción laboral, y desde ahí lograr ser profesores efectivos.

\section{MARCO TEÓRICO-REFERENCIAL}

\subsection{TRANSFORMACIONES DEL TRABAJO DOCENTE}

Reyes y Cornejo (2008), en una revisión sobre la evolución de la "cuestión docente" en Chile, muestran la intensificación del trabajo del profesor producto de un aumento en la cantidad de labores y responsabilidades durante el mismo tiempo de trabajo, como es la evaluación y aplicación de proyectos de la escuela. Junto a ello y como consecuencia de la crisis de la institución socializadora básica, la familia, deben incluir también temáticas como el uso de drogas, alcohol y sexualidad. Esta falta de tiempo para la preparación de clases, para descansos durante la jornada laboral o por la necesidad de tener más de un trabajo aparece en diferentes estudios sobre profesores chilenos, latinoamericanos y estadounidenses (Liu \& Ramsey, 2008; Robalino \& Körner, 2005; Vaillant \& Rossel, 2006). 
Una revisión de la literatura internacional de investigaciones realizadas en Chile, América Latina y otros países en desarrollo muestra un escenario complejo para la profesión docente, por la evidencia de situaciones objetivas relacionadas a salarios insuficientes, intensificación de las tareas del docente y baja autonomía profesional (Ávalos et al., 2010). Algunos factores organizaciones actúan como como barrera y dificultan la mejora escolar, como la carga de trabajo, el clima negativo de la clase, débil autonomía y de control en el trabajo y diferencias surgidas con supervisores y compañeros (Doménech, 2011). En contraposición a los obstáculos percibidos del docente, se encuentran los facilitadores del trabajo, compuestos por los facilitadores sociales (que hacen referencia a la planificación, gestión eficaz de reuniones, apoyo de los compañeros y padres), la gestión de la indisciplina (que abre la posibilidad de amonestar a estudiantes) y la gestión eficaz del aula (que permite cambiar la dinámica de las actividades de la clase) (Salanova, Martínez \& Lorente, 2005).

Además, en relación con los factores protectores y/o moduladores del malestar y estrés docente, la investigación ha mostrado de forma consistente la influencia de variables personales relacionadas al campo de la psicología positiva, las principales son el locus de control interno, la autoestima, la autoeficacia, la inteligencia emocional, la personalidad resistente y la resiliencia (Doménech, 2011).

\subsection{RESILIENCIA}

El interés por el concepto de resiliencia surge de tres áreas de investigación: a) las diferencias individuales en poblaciones de alto riesgo, b) estudios sobre temperamento y c) las diferentes formas en que los sujetos enfrentan situaciones, experiencias claves o transiciones (Losada \& Latour, 2012). La resiliencia abre la posibilidad de que no todas las personas que se han visto expuestas a situaciones críticas sufran enfermedad o cuadros psicopatológicos, sino que hay personas que superan dichas situaciones (Losada \& Latour, 2012).

Saavedra y Villalta (2008b) señalan que la respuesta resiliente es una acción orientada a metas, sustentada o vinculada a una visión abordable del problema; es una conducta recurrente que se caracteriza por elementos afectivos y cognitivos positivos o proactivos ante los problemas, los cuales tienen de base un sistema de creencias y vínculos sociales de seguridad básica de la persona.

En lo que se refiere a estudios de resiliencia en profesores, un estudio peruano no encontró diferencias significativas al comparar el nivel la resiliencia entre docentes de primaria pertenecientes a dos distritos escolares de muy distintas condiciones de trabajo (económicas, sociales, políticas, culturales y laborales), concluyéndose que la resiliencia va más allá de ser una condición dependiente de factores externos a la persona (López, 2012).

Otro estudio peruano comparó los niveles de resiliencia en 710 profesores según el nivel de enseñanza impartido (inicial, primaria y secundaria), informando que los docentes de educación inicial presentaban mayor nivel de resiliencia que sus pares de primaria y secundaria, y que estos últimos obtuvieron mayores niveles que los de primaria (Guerra, 2010).

En cuanto a estudios con profesores en el contexto nacional, Villalta y Saavedra (2012) evaluaron, por medio de cuestionarios, la resiliencia, las prácticas cotidianas de enseñanza y la cultura institucional en profesores de establecimientos de educación secundaria de sectores vulnerables con alto logro de aprendizaje. Aunque se encontró una correlación 
moderada entre prácticas cotidianas de enseñanza y cultura institucional, se observó nula correlación de ambas variables con la puntuación de la escala de resiliencia.

A modo de conclusión, la literatura muestra pocos estudios realizados sobre los niveles de resiliencia en docentes, y los existentes muestran puntos contradictorios en lo que se refiere a la relación de la resiliencia con variables demográficas. Asimismo, son puntuales los estudios que consideran las variables años de experiencia y nivel de enseñanza impartido.

\subsection{SATISFACCION LABORAL}

El concepto de satisfacción laboral es un término amplio, pudiendo encontrarse diferentes y numerosas definiciones. Al respecto, Abrajam, Contreras y Montoya (2009) refieren que la satisfacción es la concordancia entre la persona y el rol que desempeña, incluyendo dos facetas: la primera es la satisfacción intrínseca, asociada a la naturaleza del trabajo y las percepciones que el trabajador tiene del puesto que realiza, y la segunda faceta corresponde a la satisfacción extrínseca, relacionada a salario y prestaciones. Cuadra y Veloso (2007) definen la satisfacción laboral como un estado interno que es expresado por la evaluación afectiva y/o cognitiva de las experiencias de trabajo de manera favorable o desfavorable.

En el área educativa, la satisfacción laboral del profesor es importante para lograr un trabajo saludable y productivo; es el resultado de diversos factores que incluyen la evaluación que el profesor hace de su ambiente laboral. Tales factores corresponden al aspecto cognoscitivo, o sea las creencias e ideologías que el individuo tiene de su trabajo, el aspecto afectivo que dice relación con las emociones vivenciadas al trabajar y el aspecto conductual que incluye el comportamiento mismo que la persona realiza al desarrollar su rol (Linares \& Gutiérrez, 2010).

Otra de las consecuencias de que el profesorado esté satisfecho es que está en mejores condiciones de desarrollar su trabajo, por lo que es valioso promover grados adecuados de satisfacción mediante intervenciones de carácter formativo (Reyes, 2013).

En lo que respecta a estudios chilenos sobre satisfacción laboral de los docentes, un estudio en la ciudad de Rengo con 89 profesores de establecimientos municipales encontró una relación directa entre apoyo social y satisfacción laboral y una relación inversa de estas dos variables con el burnout. Se aprecia que del total de docentes, alrededor de un 55\% de estos exhibe niveles de satisfacción laboral que oscilan entre alto y muy alto (Jiménez, Jara \& Miranda, 2012). Por su parte, en el estudio de Claro y Bedregal (2003) con profesores de Puente Alto la satisfacción laboral correlacionó positivamente con la relación con directivos y con los estudiantes. Respecto a qué factores podrían aumentar la satisfacción de su labor docente, los principales eran una mayor valoración de la sociedad, aumentar las remuneraciones y mejores relaciones humanas en general.

Se puede concluir a partir de los estudios revisados que son escasas las investigaciones en docentes chilenos que incluyan la satisfacción laboral. Junto con los estudios internacionales, los hallazgos mayoritariamente muestran niveles adecuados de satisfacción laboral docente. Respecto a variables sociodemográficas relacionadas con la satisfacción docente, gran parte de los estudios concluyen que no hay diferencias significativas de satisfacción laboral en relación con la edad y el sexo, existiendo menos información respecto a la posible influencia de la variable años de experiencia y nivel educativo. No obstante, las investigaciones concuerdan en señalar que existiría una correlación negativa 
entre nivel de satisfacción laboral docente y variables relacionadas con salud mental como burnout y sintomatología depresiva.

Uno de los factores que incide en el grado de satisfacción laboral es el clima de trabajo, que en el ámbito educativo es en gran medida el resultado de las relaciones que se establecen entre colegas. Así, el grado de satisfacción afecta el comportamiento, la dedicación y conductas específicas como la colaboración y el absentismo, estableciéndose de esta forma una relación mutua y circular. Sin embargo, la relación entre clima laboral desfavorable y un estado de malestar e insatisfacción puede estar mediada por factores personales como la resiliencia del individuo que actúa como un factor de protección, atenuando o previniendo el estado de malestar (Reyes, 2013).

Un estudio de Matos et al. (2010) examinó la relación entre resiliencia y satisfacción laboral en una muestra de enfermeros y enfermeras psiquiátricos, un grupo sometido a considerable estrés en su lugar de trabajo. No se encontraron diferencias significativas en relación a la edad, sexo y años de experiencia como profesional. Se encontró una correlación de 0.33 entre ambas variables y la dimensión de resiliencia que presentaba mayor relación con la satisfacción laboral correspondía al estatus profesional, asociado a sentido de vida, crecimiento y autoestima. Por lo cual se puede pensar que la resiliencia sería una de las variables que interviene en la mejora de la satisfacción laboral y amortiguaría el estrés laboral.

En el ámbito educativo, la resiliencia podría ser una variable moduladora del malestar docente, permitiendo al profesor sobreponerse o adaptarse a situaciones estresantes. Esto tendría como consecuencias mayor dedicación, motivación y vigor en el objetivo de alcanzar el aprendizaje y responder a las demandas que exige la profesión docente (Doménech, 2011).

\section{METODO}

Este es un estudio descriptivo-correlacional, con diseño transversal en un enfoque cuantitativo que tuvo como objetivo identificar la influencia de la resiliencia sobre la satisfacción laboral en profesores pertenecientes a establecimientos municipales y particulares subvencionados de la comuna de Machalí.

\subsection{PARTICIPANTES}

El tipo de muestreo utilizado es de tipo no probabilístico por conveniencia. La muestra estuvo compuesta por 119 profesores, de ellos 80 profesores pertenecientes a 5 establecimientos municipales y 39 profesores pertenecientes a 3 colegios particulares subvencionados de la Comuna de Machalí. De los 119 profesores encuestados, 23,5\% corresponde a hombres (28), 74,2\% mujeres (89), y hubo un $1,7 \%$ que omitió su sexo (2). Tienen edades comprendidas entre 23 y 69 años, con un promedio de edad de 39,7 años.

El tamaño de la muestra se estableció a partir de la relación entre resiliencia y satisfacción laboral reportada por Matos et al. (2010) de $r=0,33$. Considerando como estimada una correlación poblacional de $\rho=0,3$, para obtener un poder de 0,8 con un nivel de significación de 0,05 se necesitaba una muestra de mínimo 85 personas. De esta forma, si se estima una pérdida muestral de un $25 \%$ y redondea a la décima más cercana, la muestra mínima correspondería a 110 personas. 
Estudios Pedagógicos XLIII, $N^{\circ}$ 1: 75-86, 2017

RESILIENCIA Y SATISFACCIÓN LABORAL EN PROFESORES DE COLEGIOS MUNICIPALES Y PARTICULARES

SUBVENCIONADOS DE LA COMUNA DE MACHALÍ

\subsection{INSTRUMENTOS}

\section{Información sociodemográfica}

Se solicitó información sociodemográfica básica, asociada a antecedentes personales como edad y sexo. Información vinculada al área profesional como número de años que se desempeña como profesor, número de años en el establecimiento educacional actual y nivel de enseñanza impartido.

\section{Escala de resiliencia SV-RES (Saavedra \& Villalta, 2008)}

Posee 60 ítems con cinco alternativas de respuesta que representan el grado en que cada afirmación describe al sujeto "Muy de acuerdo", "De acuerdo", "Ni de acuerdo ni desacuerdo", "En acuerdo" y "Muy desacuerdo", por lo cual los puntajes globales pueden variar entre 60 y 300 (Saavedra \& Villalta, 2008a).

Mide el constructo resiliencia por medio de un modelo que incluye doce categorías que se agrupan en cuatro ámbitos de profundidad y a la vez en tres modalidades de interacción: consigo mismo, con los otros y con sus posibilidades. El primer ámbito de profundidad corresponde a Condiciones de base e incluye las categorías de Identidad, Vínculos y Afectividad. El segundo ámbito de profundidad es Visión de Sí Mismo que incorpora las categorías de Autonomía, Redes y Autoeficacia. El tercer ámbito es Visión del Problema que incluye las categorías de Satisfacción, Modelos y Aprendizaje. Finalmente, el cuarto ámbito de profundidad es Respuesta Resiliente que incluye como categorías Pragmatismo, Metas y Generatividad.

Posee validez de criterio (correlación de Pearson=0.76) evaluada a través del método interprueba con la escala CD-Risc (Connor \& Davidson, 2003). Cuenta con validez de contenido apoyada por la opinión de 10 jueces conocedores de la materia y cercanos a su investigación. Durante el año 2007 el instrumento fue aplicado en tres estudios con un total de 440 personas participantes. Analizado en una población entre 15 y 65 años, cuenta con baremo para población chilena (Saavedra, 2011). Respecto a su confiabilidad por consistencia interna, en el presente estudio se obtuvo un Alfa de Cronbach de 0.96.

\section{Escala de satisfacción laboral versión para profesores ESL-VP (Anaya, 2005)}

Consta de 32 ítems, cada uno con cinco alternativas de respuesta que representan el grado en que en el actual trabajo se dan ciertas situaciones "Muy bajo", "Bajo", "Medio", "Alto" y "Muy alto", por lo cual los puntajes pueden variar entre 32 y 160 puntos. Mide el constructo satisfacción laboral de forma multidimensional, por medio de un modelo de 32 facetas que se estructuran en cinco dimensiones: diseño del trabajo, condiciones de vidas asociadas al trabajo, realización personal, promoción y superiores y salario (Anaya y Suárez, 2007).

Los coeficientes Alpha de Cronbach indican la buena consistencia interna de la escala, tanto en lo que concierne a la muestra general como a los grupos categóricos en función de la etapa educativa y la antigüedad profesional y género (muestra general .92; infantil .92: primaria .91: secundaria .92: menos de 5 años .91: entre 5 y 10 años .92: más de 10 años .92: mujeres .92: y hombres .91) (Anaya \& Suárez, 2007). En el presente estudio se obtuvo un coeficiente Alpha de Cronbach de 0.96. 


\subsection{PROCEDIMIENTO}

Para acceder a los centros educativos municipales se contactó al jefe del departamento de administración de la educación municipal de la comuna de Machalí, donde se obtuvo el permiso formal; se identificaron los establecimientos educativos existentes y el número actualizado de profesores por establecimiento. Posteriormente, se visitó cada establecimiento educativo, coordinándose con el director el proceso de aplicación.

Paralelamente, para acceder a los establecimientos particulares subvencionados de la Comuna de Machalí, se visitó personalmente cada colegio para obtener el permiso con el director, previa presentación de la investigación.

La aplicación de los instrumentos se efectuó previa coordinación de día y hora para concretar el trabajo de campo que fue de aproximadamente cinco meses. Específicamente se realizó una explicación inicial manifestando la intencionalidad y trascendencia del estudio, solicitando la voluntad de participar. Se entregó individualmente el consentimiento informado, dando a conocer la confidencialidad de la información. El tiempo estimado de la aplicación de instrumentos a los profesores fue una sesión de 25 minutos aproximadamente como máximo. Al finalizar el proceso, se agradeció al grupo de profesores la participación en el estudio.

\subsection{ANÁLISIS ESTADÍSTICO}

Se utilizó para el análisis y manejo de los datos el software SPSS para Windows. Se determinó la confiabilidad de las escalas por medio del coeficiente Alfa de Cronbach, se calcularon los estadísticos descriptivos de las distintas variables, se determinaron los coeficientes de correlación de Pearson entre las variables principales y, finalmente, se realizó una regresión lineal para identificar el grado en que las variables independientes predicen la variable dependiente.

\section{RESULTADOS}

En primer lugar se presentan los estadísticos descriptivos generales de las variables estudiadas.

Tabla 1. Estadísticos descriptivos de las distintas variables

\begin{tabular}{|l|c|c|c|c|c|}
\hline & Puntaje mínimo & Puntaje máximo & Mediana & Media & DE \\
\hline Edad & 23 & 69 & 37 & 39.7 & 11.9 \\
\hline Antigüedad docente & 1 & 50 & 9 & 13.7 & 11.9 \\
\hline Años en colegio & 1 & 40 & 4 & 7 & 8.4 \\
\hline Resiliencia & 216 & 295 & 277 & 269.2 & 20.3 \\
\hline Satisfacción laboral & 54 & 156 & 106 & 107.3 & 22.2 \\
\hline
\end{tabular}


En cuanto a las variables principales del estudio, se observa en la Tabla 1 que el nivel de resiliencia de los participantes es alto, ya que el puntaje promedio del grupo (269.2) representa un $89,7 \%$ del puntaje máximo posible (300). En cambio, el nivel de satisfacción laboral es menor, ya que el puntaje promedio del grupo (107.3) representa solo un $67 \%$ del puntaje máximo posible (160).

A continuación se presentan los coeficientes de correlación de Pearson entre las dos variables en estudio y características socio-demográficas de la muestra.

Tabla 2. Correlaciones entre las variables

\begin{tabular}{|l|c|c|}
\hline & Resiliencia & Satisfacción laboral \\
\hline Edad & 0,10 & $0,19 *$ \\
\hline Antigüedad docente & 0,15 & $0,28 *$ \\
\hline Años en colegio & 0,04 & 0,08 \\
\hline Resiliencia & - & $0,43 * *$ \\
\hline Satisfacción laboral & $0,43 * *$ & - \\
\hline
\end{tabular}

Tal como se observa en la Tabla 2, existe una relación significativa entre el nivel de resiliencia de los participantes y su nivel de satisfacción laboral. El nivel de resiliencia no presenta relaciones significativas con la edad ni con los años de ejercicio docente en el mismo colegio, aunque se puede apreciar una relación positiva con la antigüedad docente que no alcanza a ser estadísticamente significativa para el tamaño de la muestra de este estudio. En cambio, el nivel de satisfacción laboral de los participantes presenta relaciones positivas significativas tanto con su antigüedad docente como con su edad.

Por último, la Tabla 3 muestra los coeficientes de regresión lineal de las variables edad, antigüedad docente y resiliencia sobre la variable dependiente satisfacción laboral.

Tabla 3. Coeficientes de regresión de la edad, la antigüedad docente y la resiliencia sobre la satisfacción laboral

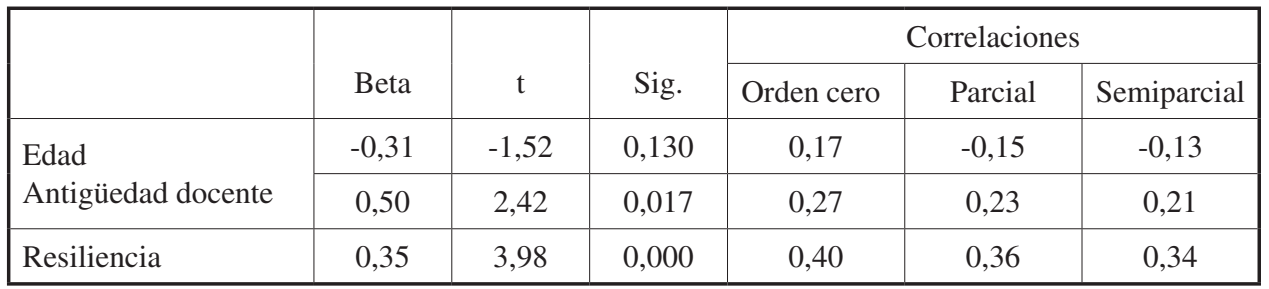

Variable dependiente: satisfacción laboral. Fuente: elaboración propia. 
Se puede observar que tanto el nivel de resiliencia como la antigüedad docente influyen positivamente de manera significativa sobre la variable satisfacción laboral. Es decir, aquellos participantes que muestran mayor nivel de resiliencia o que tienen más años de ejercicio docente es más probable que presenten mayor satisfacción laboral. En cambio la variable edad no tendría una influencia significativa sobre la variable satisfacción laboral en esta muestra.

\section{DISCUSIÓN}

Un primer aspecto a destacar en los resultados de este estudio es que el nivel reportado por los docentes para la variable resiliencia es bastante alto, lo cual permite tener una mirada muy positiva acerca de las condiciones personales de los docentes, necesarias para enfrentar los desafíos crecientes de su labor profesional. En cambio los niveles reportados de satisfacción laboral de la muestra son menores a los de resiliencia y corresponden a un nivel intermedio, lo cual concuerda con un estudio en profesores mexicanos donde la satisfacción laboral se posicionó en el punto medio (Martínez, 2007). Este resultado difiere de la satisfacción laboral relativamente alta reportada en otros estudios con profesores mexicanos (Linares \& Gutiérrez, 2010), colombianos (Hermosa, 2006) y chilenos (Jiménez et al., 2012).

La relación positiva significativa entre la variable resiliencia y la satisfacción laboral en este grupo de profesores, confirmada ampliamente en el análisis de regresión, concuerda con lo encontrado en un estudio que abordó estas dos variables en una muestra de enfermeras psiquiátricas (Matos et al., 2010). Ello indicaría que las características disposicionales de la resiliencia serían factores que permiten que en alguna medida los profesores deriven satisfacción de su desempeño laboral a pesar del complejo escenario en el cual desempeñan su rol docente. Este escenario complejo incluye la intensificación del trabajo, falta de tiempo y descansos en la jornada laboral, falta de recursos, sensación de abandono, culpabilización (Liu \& Ramsey, 2008; Robalino \& Körner, 2005; Vaillant \& Rossel, 2006), y también guarda relación con estresores percibidos intensamente por docentes (Manassero et al., 2005), obstáculos técnicos, sociales y de salud mental (Salanova et al., 2005), además de los riesgos psicosociales presentes en cualquier tipo de organización, sobre todo en el sector servicios (Quiceno \& Vinaccia, 2007).

Lo anterior resume la percepción de la situación compleja que vive el profesor, sin embargo los profesores participantes del presente estudio no presentan un bajo nivel de satisfacción laboral. Ello podría ser explicado considerando la relación significativa entre la satisfacción laboral y la resiliencia, estando esta última variable en un nivel relativamente alto. Por lo que esta variable independiente sería un factor que permite que los profesores mejoren la percepción de bienestar asociado a la satisfacción laboral. Además, no todos los profesores se verían afectados por las condiciones adversas en que desempeñan su rol, ya que su nivel de resiliencia estaría actuando como un amortiguador del efecto de los eventos negativos (Fiorentino, 2008), permitiéndoles sobreponerse a los obstáculos y conseguir sus metas (Saavedra, 2011).

El hecho de que la variable resiliencia no haya presentado relación significativa con la edad concuerda con un estudio mexicano efectuado en adultos (González \& Valdez, 2011), en cambio difiere de lo reportado por Saavedra y Villalta (2008b), quienes encontraron un mayor nivel de resiliencia en adultos jóvenes (19-24 años) que en adultos medios (46-55 
años), diferencia que podría explicarse por los distintos rangos de edad de los participantes en ambos estudios. De esta manera se podría señalar que en este grupo de profesores la capacidad de superar situaciones críticas donde la persona no se desanima y no se deja vencer (Losada \& Latour, 2012) va más allá de la edad, así como de la antigüedad y años de ejercicio docente, constituyendo de esta forma la resiliencia una base de creencias que impregna la seguridad de la persona y las interpretaciones que realiza (Saavedra \& Villalta, 2008b).

El análisis de regresión lineal mostró que, además de la resiliencia, la otra variable que tiene influencia sobre la satisfacción laboral es la antigüedad docente. Ello se podría explicar porque mientras más experiencia tienen las personas desempeñando un rol pueden derivar más satisfacción de tal desempeño (Abrajam et al., 2009).

Si consideramos la resiliencia como una conducta recurrente vinculada a una mirada abordable de los problemas, que se basa en aspectos afectivos y cognitivos positivos fundados en las creencias y vínculos de las personas (Saavedra \& Villalta 2008b), podríamos señalar que esta visión proactiva es la que comparten el grupo de profesores estudiados, constituyendo una característica clave para la satisfacción laboral percibida y un factor que resultaría protector en la percepción subjetiva de la experiencia que los profesores tienen de su trabajo, y es a la vez un variable moduladora del malestar docente (Domenech, 2011).

Se sugiere para futuras investigaciones realizar estudios prospectivos que permitan determinar con mayor exactitud el valor predictivo de las variables independientes estudiadas. Además, para enriquecer el conocimiento sobre la satisfacción laboral de los profesores se sugiere incluir nuevas variables independientes. Finalmente, sería deseable incluir otras variables socioculturales para comparar los resultados con investigaciones de otros medios y así obtener mayores explicaciones de los hallazgos obtenidos.

\section{REFERENCIAS BIBLIOGRÁFICAS}

Abrajan, M., Contreras, J., \& Montoya, S. (2009). Grado de satisfacción laboral y condiciones de trabajo: una exploración cualitativa. Enseñanza e Investigación en Psicología, 14(1), 105-118.

Anaya, D., \& Suárez. J. (2007). Satisfacción laboral de los profesores de educación infantil, primaria y secundaria. Un estudio de ámbito nacional. Revista de Educación, 344, 217-243.

Arancibia, V., Herrera, P., \& Strasser, K. (2008). Manual de Psicología Educacional (6a ed.). Santiago de Chile: Ediciones Universidad Católica de Chile.

Ávalos, B., Cavada, P., Pardo, M., \& Sotomayor, C. (2010). La profesión docente: temas y discusiones en la literatura internacional. Estudios Pedagógicos, 36(1), 235-263.

Cuadra, A., \& Veloso, C. (2007). Liderazgo, clima y satisfacción laboral en las organizaciones. Revista Universum, 2(22), 42-58.

Claro, S., \& Bedregal, P. (2003). Aproximación al estado de salud mental del profesorado en 12 escuelas de Puente Alto, Santiago, Chile. Revista Médica de Chile, 131, 159-167.

Connor, K., \& Davidson, J. (2003). Development of a new resilience scale: the Connor-Davidson Resilience Scale (CD-RISC). Depression \& Anxiety, 18(2), 76-82.

Corvalán, M.I. (2005). La realidad escolar cotidiana y la salud mental de los profesores. Revista Enfoques Educacionales, 7(1), 69-70.

Doménech, D. (2011). El bienestar psicológico del profesorado: variables implicadas. Anuari de l'Agrupació Borrianenca de Cultura, 22, 27-40. Recuperado de http://www3.uji.es/ betoret/ Investigacion/BORRADOR\%2012\%20ARTICULO $\% 20$ con\%20resumen\%20y\%20pie.pdf

Fiorentino, M.T. (2008). La construcción de la resiliencia en el mejoramiento de la calidad de vida y la salud. Suma Psicológica, 15(1), 95-114. 
González, N., \& Valdés, J. (2011). Resiliencia y personalidad en adultos. Revista Electrónica de Psicología Iztacala, 14(4), 295-316.

Guerra, J. (2010). Los niveles de resiliencia en los docentes de Inicial, Primaria y Secundaria de la Región Callao (Tesis para optar el grado académico de Magíster en Educación). Universidad San Ignacio de Loyola, Lima, Perú. Recuperado de http://repositorio.usil.edu.pe/wp-content/ uploads/2014/07/2010_Guerra_Niveles-de-resiliencia-en-docentes-de-inicial-primaria-ysecundaria-de-la-Regi\%C3\%B3n-Callao.pdf

Hermosa, M. A. (2006). Satisfacción laboral y síndrome de "Burnout" en profesores de educación primaria y secundaria. Revista Colombiana de Psicología, 15(1), 81-89.

Jiménez, A., Jara M.J., \& Miranda E. (2012). Burnout, apoyo social y satisfacción laboral en docentes. Revista Semestral da Associação Brasileira de Psicologia Escolar e Educacional, SP, 16(1), 125-134.

Linares, O., \& Gutiérrez, R. (2010). Satisfacción laboral y percepción de salud mental en profesores. Revista Mexicana de Investigación en Psicología, 2(1), 31-36.

Liu, X. S., \& J. Ramsey (2008). Teachers' job satisfaction: Analyses of the Teacher Follow-up Survey in the United States for 2000-200. Teaching and Teacher Education, 24(5), 1115-1400.

López, I. (2012). Niveles de resiliencia en los docentes de primaria de las instituciones públicas de Ventanilla y Callao (Tesis para optar el grado académico de Maestro en Educación). Universidad San Ignacio de Loyola, Lima, Perú. Recuperado de http://repositorio.usil.edu.pe/wpcontent/ uploads/2014/07/2012_L\%C3\%B3pez_Niveles-de-resiliencia-en-los-docentes-de-primaria-delas-instituciones-p\%C3\%BAblicas-de-Ventanilla-y-Callao.pdf

Losada, V., \& Latour, M.I. (2012). Resiliencia. Conceptualización e investigación en Argentina. Psiencia Revista Latinoamericana de Ciencia Psicológica, 4(2) 84-97.

Manassero, M., García, E., Torres, G., Ramis, C., Vásquez, A., \& Ferrer, V.A. (2005). Burnout en la enseñanza: aspectos atribucionales. Revista de Psicología del Trabajo y de las Organizaciones, 21(1-2), 55-70.

Martínez, O. (2007). El desarrollo profesional de los docentes de secundaria: incidencia de algunas variables personales y de actuación profesional (Tesis de Doctorado, Universidad Autónoma de Barcelona, España). Recuperado de http://www.tdx.cat/bitstream/handle/10803/5048/oemt1de1. pdf;jsessionid=37853D79E72D7F1EAB48B8DCA10E18DD.tdx2? sequence=1

Matos, P., Neushotz, L., Quinn, M., \& Fitzpatrick, J. (2010). An exploratory study of resilience and job satisfaction among psychiatric nurses working in impatient units. International Journal of Mental Health Nursing, 19, 307-312.

Quiceno, J., \& Vinaccia, S. (2007). Burnout: síndrome de quemarse en el trabajo. Acta Colombiana de Psicología, 10, 117-125.

Reyes, M. (2013). Del malestar al compromiso: los factores individuales en la satisfacción laboral de los docentes. Revista Inter-Ação, 38(1), 37-50. Recuperado de http://revistas.ufg.emnuvens. com.br/interacao/article/view/18736/15118

Reyes, L., \& Cornejo, R. (2008). La cuestión docente en América Latina. Estudio de caso: Chile. Santiago: Foro Latinoamericano de Políticas Educativas. Recuperado de http://www.opech.cl/ inv/investigaciones/FLAPE\%20FINAL\%20Y\%20ENVIADO.pdf

Robalino, M., \& A. Körner. (2005). Condiciones de trabajo y salud docente. Condiciones de trabajo y salud docente. Estudios de casos en Argentina, Chile, Ecuador, México, Perú y Uruguay. Santiago: UNESCO. Recuperado de http://unesdoc.unesco.org/images/0014/001425/142551s.pdf

Saavedra, E. (2011). La resiliencia desde una mirada post racionalista. Madrid: Académica Española. Saavedra, E., \& Villalta, M. (2008a). Escala de Resiliencia SV-RES, para jóvenes y adultos. Santiago: CEANIM.

Saavedra, E., \& Villalta, M. (2008b). Medición de las características resilientes, un estudio comparativo en personas entre 25 y 64 años. Liberabit, 14, 31-40.

Salanova, M., Martínez, I.M., \& Lorente, L. (2005). ¿Cómo se relacionan los obstáculos y facilitadores 
Estudios Pedagógicos XLIII, $\mathrm{N}^{\circ}$ 1: 75-86, 2017

RESILIENCIA Y SATISFACCIÓN LABORAL EN PROFESORES DE COLEGIOS MUNICIPALES Y PARTICULARES

SUBVENCIONADOS DE LA COMUNA DE MACHALÍ

organizacionales con el burnout docente? Un estudio longitudinal. Revista de Psicología del Trabajo y de las Organizaciones, 21(1-2), 37-54.

Vaillant, D., \& C. Rossel. (2006). Maestros de Escuelas Básicas en América Latina. Hacia una radiografía de la profesión. Santiago: PREAL. Recuperado de http://www.oei.es/docentes/ publicaciones/maestros_escuela_basicas_en_america_latina_preal.pdf

Villalta, M., \& Saavedra, E. (2012). Cultura escolar, prácticas de enseñanza y resiliencia en alumnos y profesores de contextos sociales vulnerables. Universitas Psychologica, 11(1), 67-78. 